\title{
The impact of delayed time to first CT head on functional outcomes after blunt head trauma with moderately depressed GCS
}

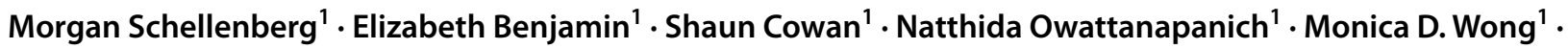 \\ Kenji Inaba ${ }^{1} \cdot$ Demetrios Demetriades $^{1}$
}

Received: 16 December 2020 / Accepted: 17 April 2021 / Published online: 14 May 2021

○) Springer-Verlag GmbH Germany, part of Springer Nature 2021

\begin{abstract}
Purpose Recent work suggests patients with moderately depressed Glasgow Coma Scale (GCS) score in the Emergency Department (ED) who do not undergo immediate head CT (CTH) have delayed neurosurgical intervention and longer ED stay. The present study objective was to determine the impact of time to first CTH on functional neurologic outcomes in this patient population.

Methods Blunt trauma patients presenting to our Level I trauma center (11/2015-10/2019) with first ED GCS 9-12 were retrospectively identified and included. Transfers and those with extracranial AIS $\geq 3$ were excluded. The study population was stratified into Immediate ( $\leq 1 \mathrm{~h}$ ) and Delayed (1-6 h) CTH groups based on time from ED arrival to first CTH. Outcomes included functional outcomes at hospital discharge based on the Modified Rankin Scale (mRS).

Results After exclusions, 564 patients were included: 414 (73\%) with Immediate CTH and 150 (27\%) Delayed CTH. Both groups arrived with median GCS 11 and alcohol/drug intoxication did not differ $(p>0.05)$. AIS Head/Neck was comparable (3[3-4] vs. 3[3-3], $p=0.349$ ). Time to ED disposition decision and ED exit were significantly shorter after Immediate CTH $(2.8[1.5-5.3]$ vs. $5.2[3.6-7.5] \mathrm{h}, \mathrm{p}<0.001$ and $5.5[3.3-8.9]$ vs. 8.1[5.2-11.7]h, $p<0.001)$. Functional outcomes were slightly worse after Immediate CTH (mRS 2[1-4] vs. 2[1-3], $p=0.002$ ). Subgroup analysis of patients requiring neurosurgical intervention demonstrated a greater proportion of moderately disabled patients with a lower proportion of severely disabled or dead patients after Immediate CTH as compared to Delayed CTH (51 vs. $20 \%, p=0.063$ and 35 vs. $60 \%, p=0.122$ ).

Conclusions Immediate CTH shortened time to disposition decision out of the ED and ED exit. Patients requiring neurosurgical intervention after Immediate CTH had improved functional outcomes when compared to those undergoing Delayed CTH. These differences did not reach statistical significance in this single-center study and, therefore, a large, multicenter study is the next step in demonstrating the potential functional outcomes benefit of Immediate CTH after blunt head trauma.
\end{abstract}

Keywords Traumatic brain injury · Time to intervention · Computed tomography of the head · Undertriage · Glasgow Coma Scale score

\section{Background}

Trauma undertriage is an important quality improvement metric in the care of severely injured trauma patients. The American College of Surgeons Committee on Trauma (ACS COT) specifies trauma team activation (TTA) criteria

Morgan Schellenberg

morgan.schellenberg@med.usc.edu

1 Division of Trauma and Surgical Critical Care, LAC + USC Medical Center, University of Southern California, 2051 Marengo Street, Inpatient Tower, C5L100, Los Angeles, CA 90033, USA which are applied prehospital to provide early identification of trauma patients who may benefit from hospital resource and personnel mobilization in advance of patient arrival [1]. When patients are severely injured but do not meet ACS COT TTA criteria, they are deemed to be undertriaged. Literature is accruing to delineate the undertriaged population [2-4] and the resultant effects of being undertriaged, including an increased risk of mortality $[5,6]$.

Recent literature suggests that undertriaged blunt trauma patients with moderately depressed Glasgow Coma Scale (GCS) score in the emergency department (ED) are at particular risk of adverse outcomes $[3,7]$. Despite being noncomatose, patients with moderately depressed GCS are at 
risk of further neurologic deterioration and must be carefully evaluated [8], with GCS in the Emergency Department (ED) predicting outcomes after traumatic brain injury (TBI) [9, 10]. A prehospital GCS score of $<9$ is required to trigger highest level TTA under existing criteria [1], and therefore patients with GCS of 9-12 do not meet the criteria for highest level TTA based on mental status alone. The adverse outcomes following undertriage of patients with moderately depressed GCS may be related to delayed times to investigations and interventions, such as computed tomography scan of the head (CTH) and neurosurgical interventions such as craniotomy or intracranial pressure (ICP) monitor insertion [7]. TTA includes expedited access to CT scan and because TBI diagnosis and care is predicated on cross-sectional imaging $[11,12]$, time to CTH may be a rate-limiting step in the care of these patients.

Although it has been established that delayed time to first $\mathrm{CTH}$ prolongs time to neurosurgical intervention and time spent in the ED [7], the impact of time to first CTH on functional neurologic outcomes is unknown. The objective of this study was to define the effect of time to first CTH on functional neurologic outcomes at hospital discharge, as quantified by the Modified Rankin Scale (mRS) score $[13,14]$, among patients with a moderately depressed GCS (9-12) on arrival to the ED after blunt trauma. The hypothesis was that delayed time to first CTH would be associated with worse neurologic outcomes at hospital discharge.

\section{Methods}

This is a single-center retrospective observational study performed at an ACS-verified, Level 1 trauma center between November 1, 2015 and October 31, 2019. Patients were identified from the trauma registry based on first ED GCS. All patients who arrived to the ED with an initial GCS of 9-12 were included. Patients were excluded if they were transferred from an outside hospital, had severe extracranial injuries (defined by an Abbreviated Injury Scale [AIS] score in the spine, chest, abdomen, or extremities of $\geq 3$ ), were injured by penetrating trauma, did not undergo CTH, or underwent first $\mathrm{CTH}>6 \mathrm{~h}$ after arrival. At our institution, trauma patients arrive as one of two levels of activation: TTA, which is triggered if patients meet standard ACS COT TTA criteria and/or are aged $>70$ years with a traumatic mechanism of injury; and routine trauma consults. Institutional Review Board approval was granted with a waiver of informed consent due to the retrospective observational nature of the study.

Data variables examined included patient demographics (age, gender, and race/ethnicity), alcohol and drug intoxication upon ED arrival, clinical data (prehospital and first ED vital signs [systolic blood pressure, SBP in $\mathrm{mmHg}$; heart rate, $\mathrm{HR}$ in beats per minute; and GCS]), and injury data (mechanism of injury, level of trauma team activation, and AIS by body region).

Study groups were defined by time from ED arrival to first CTH: Immediate ( $\leq 1 \mathrm{~h})$ vs. Delayed $(1-6 \mathrm{~h})$. These time periods were selected on the basis of existing literature for consistency and ease of comparison [7]. The primary outcome was functional neurologic status at hospital discharge, quantified by the mRS score $[13,14]$. The mRS score is numerical and ranges from 0 , which is the best score and indicates a complete lack of symptoms of neurologic impairment, to 6, which is the worst score and signifies death after neurologic disability. Secondary outcomes included time to neurosurgical intervention (craniotomy or intracranial pressure [ICP] monitor insertion); time to ED discharge disposition decision; ED length of stay (LOS), hours; hospital LOS, days; intensive care unit (ICU) LOS, days; ventilator days; blood transfusion requirements; and in-hospital mortality.

Univariate analysis compared demographics, clinical data, and injury data between study groups. Continuous variables are presented as median [interquartile range, IQR] and compared using the Mann-Whitney U test. Categorical variables are given as number (percentage) and compared using the chi-squared test. The author collecting mRS scores was blinded to the exposure of interest (time to first CTH). Subgroup analysis of only patients requiring neurosurgical intervention was planned a priori as these patients theoretically would benefit the most from prompt diagnosis with crosssectional imaging to expedite the necessary interventions.

Multivariate analysis with logistic regression was used to examine independent factors associated with in-hospital mortality. Clinically relevant variables as well as those that differed by $p<0.2$ on univariate analysis were included in the multivariate model. Only variables with a variance inflation factor (VIF) $<2.5$ were retained. Model fit was assessed using the Hosmer-Lemeshow test, with a result $>0.05$, indicating good fit. Results of the multivariate analyses are presented as odds ratios (OR) with $95 \%$ confidence intervals (CI). Statistical significance was defined as $p<0.05$. Data were collected and analyzed using IBM SPSS Statistics 23 (IBM Corporation; Armonk, NY).

\section{Results}

A total of 19,095 trauma patients presented over the study period (Fig. 1). Of these, 792 patients arrived to the ED with a GCS of 9-12 after blunt trauma. After exclusions, 564 patients $(71 \%)$ remained for analysis. Most underwent Immediate CTH $(n=414,73 \%)$, with 150 patients (27\%) undergoing Delayed CTH. Demographic, clinical, and injury data are delineated in Table 1 . Patients in both the Immediate and Delayed CTH groups arrived with 
Fig. 1 Flow of patients through study. GCS, Glasgow Coma Scale score. AIS, Abbreviated Injury Scale score. OSH, outside hospital. CTH, computed tomography scan of the head. $E D$ emergency department. Immediate $\mathrm{CTH}, \leq 1 \mathrm{~h}$ after $\mathrm{ED}$ arrival. Delayed CTH, $1-6 \mathrm{~h}$ after ED arrival

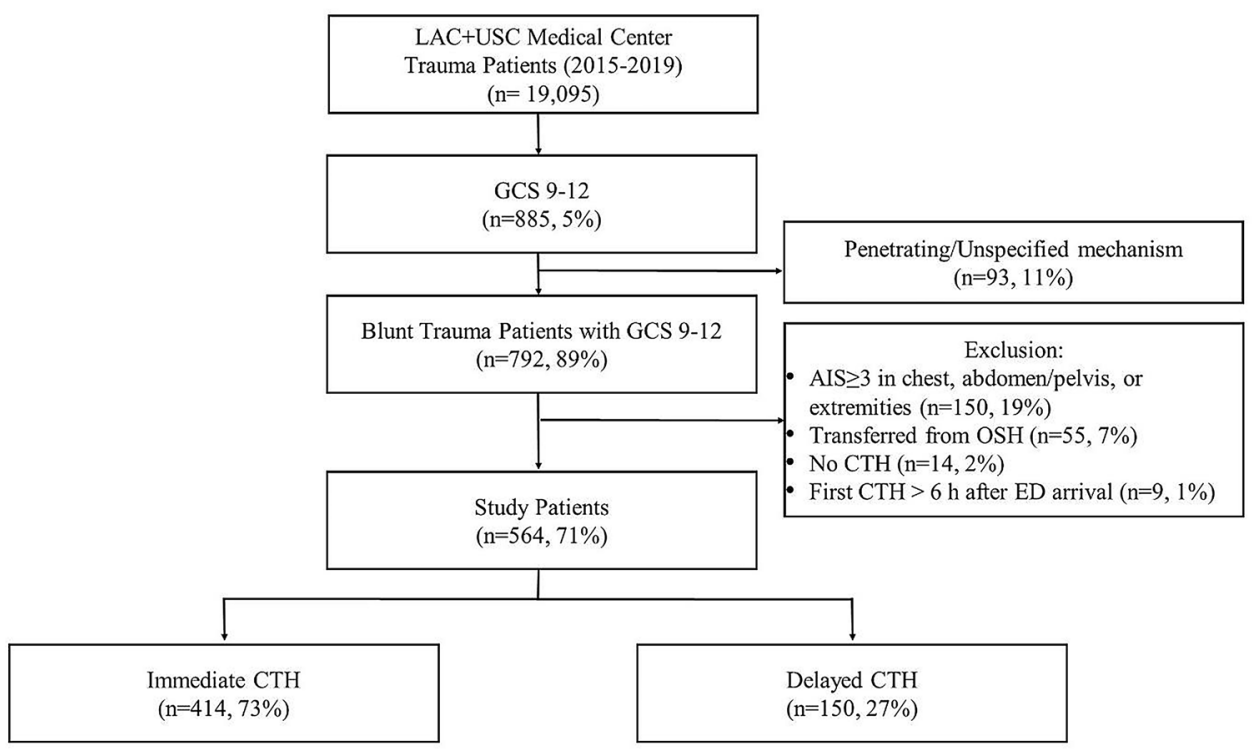

a median ED GCS of 11. Motor GCS was also similar between study groups $(5[4,5]$ vs. $5[5], p=0.677)$. AIS Head/Neck was comparable between groups $(3[3,4]$ vs. 3 [3-5], $p=0.349)$. There were no significant differences in alcohol or drug intoxication $(p>0.05)$.

Immediate $\mathrm{CTH}$ patients underwent first $\mathrm{CTH}$ at a median of 0.4 [0.3-0.6] hours after ED arrival, compared to 2.1 [1.1-2.9] hours in the Delayed CTH group $(p<0.001)$ (Table 2). Time to craniotomy (2.6 [1.6-6.4] vs. 4.0 [2.2-5.8] hours after ED arrival) and ICP monitor insertion (4.5 [2.7-9.1] vs. 8.6 [4.2-17.3] hours) were shorter in the Immediate CTH group, although these differences were not statistically significant $(p=0.313$ and $p=0.183$, respectively). Time from ED arrival to ED disposition decision and ED exit were significantly shorter among patients undergoing Immediate CTH (2.8 [1.5-5.3] vs. 5.2 [3.6-7.5] hours and 5.5 [3.3-8.9] vs. $8.1[5.2-11.7]$ hours, both $p<0.001$ ).

Mortality was comparable between groups ( 9 vs. $6 \%$, $p=0.337$ ), with a higher craniotomy rate among Immediate CTH patients (12 vs. 5\%, $p=0.023$ ) (Table 2). On multivariate analysis of in-hospital mortality, Immediate $\mathrm{CTH}$ was not independently associated with mortality (OR 1.303, $p=0.749$ ).

In terms of functional neurologic outcomes, mRS was slightly worse among Immediate CTH patients $(2[1-4]$ vs. 2 [1-3], $p=0.002$ ) (Table 1). Subgroup analysis of only patients who required neurosurgical intervention revealed comparable median MRS scores between Immediate and Delayed CTH groups (4 [3-6] vs. 5 [3-6], $p=0.451$ ) (Table 3). Immediate CTH patients had a greater proportion of patients with moderate $\mathrm{mRS}$ scores at discharge (51 vs. $20 \%, p=0.063$ ) with a lower proportion of patients who were severely disabled or dead ( 35 vs. $60 \%, p=0.122)$ when compared to Delayed CTH patients, although these differences were not statistically significant (Table 3).

\section{Discussion}

The clinical trajectory of recovery after traumatic brain injuries can be unpredictable. Some patients arriving with moderate neurologic perturbations may deteriorate while others may improve and be discharged directly home $[8,9$, 15]. Increased evidence about which interventions for these patients have a meaningful impact on outcomes is critical to improve care.

The Modified Rankin Scale (mRS) is a reliable and valid tool to quantify functional disability following neurologic insults [14], including head trauma [13], stroke [16], and anoxic brain injury following cardiac arrest [17]. To improve understanding about the impact of time to $\mathrm{CTH}$ on outcomes after TBI, the current study endeavored to use the mRS to determine if expedited time to first CTH is associated with improved functional neurologic outcomes among blunt trauma patients with an initial GCS of 9-12.

In this study, the majority of GCS 9-12 patients in both groups presented after falls as the mechanism of injury. This may imply that moderate perturbations in GCS are more common with falls than with higher energy mechanisms such as auto vs. pedestrian or motor vehicle collisions. Overall mortality in this series was low and was not impacted by time to first CTH. We suspect this is because in-hospital mortality may not be an optimal outcome metric to convey severity of a traumatic brain injury, due to the nuanced way in which head injuries evolve over time. Functional neurologic outcomes, on the other hand, may better capture the spectrum of recovery after brain injury. 
Table 1 Patient demographics, clinical data, and injury data

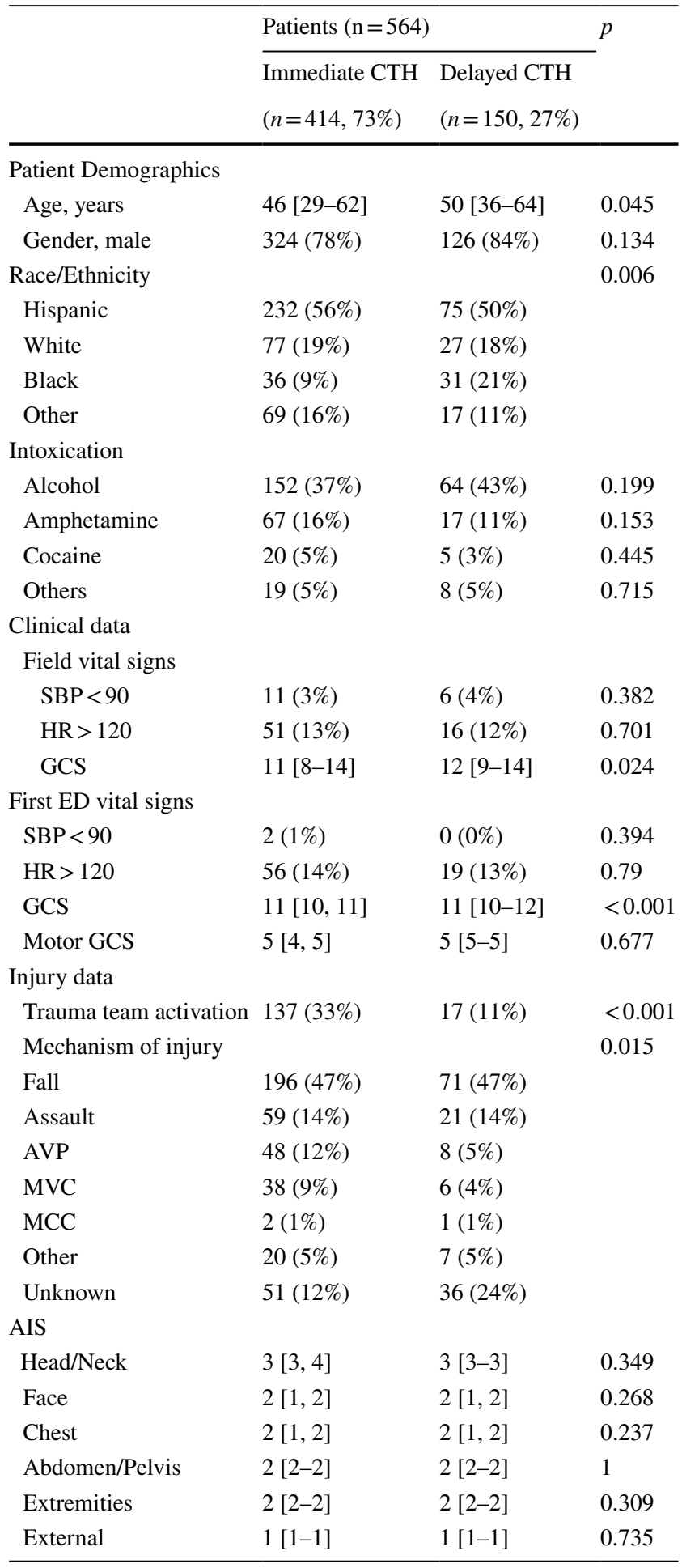

Continuous variables are presented as median [interquartile range]. Categorical variables are presented as number (percentage)

$C T H$ computed tomography scan of the head, SBP systolic blood pressure $(\mathrm{mmHg}), H R$ heart rate (beats per minute), GCS Glasgow Coma Scale score, AVP auto vs. pedestrian collision, $M V C$ motor vehicle collision, $M C C$ motorcycle collision. Other, comprised of sports/recreational injuries, crush injuries, and unspecified other mechanisms of injury, AIS, Abbreviated Injury Scale score
Table 2 Univariate analysis of outcomes

\begin{tabular}{|c|c|c|c|}
\hline & \multicolumn{2}{|l|}{ Patients $(n=564)$} & \multirow[t]{2}{*}{$p$} \\
\hline & $\begin{array}{l}\text { Immediate CTH } \\
(n=414,73 \%)\end{array}$ & $\begin{array}{l}\text { Delayed CTH } \\
(n=150,27 \%)\end{array}$ & \\
\hline \multicolumn{4}{|l|}{ Times } \\
\hline \multicolumn{4}{|l|}{ ED Arrival To: } \\
\hline First CTH & $0.4[0.3-0.6]$ & $2.1[1.1-2.9]$ & $<0.001$ \\
\hline Craniotomy & $2.6[1.6-6.4]$ & $4.0[2.2-5.8]$ & 0.313 \\
\hline ICP Monitor Insertion & $4.5[2.7-9.1]$ & $8.6[4.2-17.3]$ & 0.183 \\
\hline $\begin{array}{l}\text { ED disposition deci- } \\
\text { sion }\end{array}$ & $2.8[1.5-5.3]$ & $5.2[3.6-7.5]$ & $<0.001$ \\
\hline ED Exit & $5.5[3.3-8.9]$ & $8.1[5.2-11.7]$ & $<0.001$ \\
\hline \multicolumn{4}{|l|}{ First CTH To: } \\
\hline Craniotomy & $2.0[1.1-6.0]$ & $1.4[0.9-1.8]$ & 0.347 \\
\hline ICP Monitor Insertion & $4.1[2.3-8.6]$ & $6.5[1.5-15.8]$ & 0.861 \\
\hline $\begin{array}{l}\text { ED disposition deci- } \\
\text { sion }\end{array}$ & $2.2[0.9-4.8]$ & $3.2[1.3-5.7]$ & 0.012 \\
\hline ED Exit & $5.0[2.7-8.3]$ & $6.2[3.3-9.9]$ & 0.012 \\
\hline \multicolumn{4}{|c|}{ Modified Rankin Scale Score } \\
\hline Median & $2[1-4]$ & $2[1-3]$ & 0.002 \\
\hline $0-2$ & $241(58 \%)$ & $105(70 \%)$ & 0.011 \\
\hline 4-Mar & $126(30 \%)$ & $35(23 \%)$ & 0.099 \\
\hline 6-May & $47(12 \%)$ & $10(7 \%)$ & 0.103 \\
\hline Mortality & $35(9 \%)$ & $9(6 \%)$ & 0.337 \\
\hline $\begin{array}{l}\text { Craniotomy/Craniec- } \\
\text { tomy }\end{array}$ & $49(12 \%)$ & $8(5 \%)$ & 0.023 \\
\hline ICP Monitor & $58(14 \%)$ & $9(6 \%)$ & 0.009 \\
\hline ED Intubation & $110(27 \%)$ & $24(16 \%)$ & 0.009 \\
\hline \multicolumn{4}{|l|}{ Blood transfusion, any } \\
\hline First $4 \mathrm{~h}$ & $20(5 \%)$ & $1(1 \%)$ & 0.021 \\
\hline First $24 \mathrm{~h}$ & $32(8 \%)$ & $4(3 \%)$ & 0.03 \\
\hline During Hospitalization & $71(17 \%)$ & $15(10 \%)$ & 0.037 \\
\hline Hospital LOS, days & $10[5-25]$ & $8[6-18]$ & 0.004 \\
\hline ICU LOS, days & $5[3-15]$ & $5[3-11]$ & 0.113 \\
\hline Ventilator Days & $3[2-8]$ & $2[2-5]$ & 0.028 \\
\hline
\end{tabular}

Continuous variables are presented as median [interquartile range]. Categorical variables are presented as number (percentage)

$C T H$, computed tomography scan of the head. Times, in hours. ED emergency department, ICP intracranial pressure, $h$ hours, LOS length of stay (days), ICU intensive care unit

We identified an improvement in functional outcomes overall among patients who underwent Delayed CTH when compared to those undergoing Immediate CTH. The most likely explanation is that patients who underwent Delayed CTH were less critically injured than patients who underwent Immediate CTH, as suggested by the interval of time permitted to elapse prior to cross-sectional imaging. Conversely, we also identified that functional outcomes were better after Immediate CTH than after Delayed CTH when subgroup analysis was performed of only patients who 
Table 3 Univariate analysis of outcomes among patients requiring neurosurgical intervention

\begin{tabular}{|c|c|c|c|}
\hline & \multicolumn{2}{|l|}{ Patients $(n=82)$} & \multirow[t]{2}{*}{$p$} \\
\hline & $\begin{array}{l}\text { Immediate CTH } \\
(n=72)\end{array}$ & $\begin{array}{l}\text { Delayed CTH } \\
(n=10)\end{array}$ & \\
\hline \multicolumn{4}{|c|}{ Modified Rankin Scale Score } \\
\hline Median & $4[3-6]$ & $5[3-6]$ & 0.451 \\
\hline $0-2$ & $10(14 \%)$ & $2(20 \%)$ & 0.608 \\
\hline $3-4$ & $37(51 \%)$ & $2(20 \%)$ & 0.063 \\
\hline $5-6$ & $25(35 \%)$ & $6(60 \%)$ & 0.122 \\
\hline Mortality & $19(26 \%)$ & $5(50 \%)$ & 0.124 \\
\hline Need for Craniotomy & $49(69 \%)$ & $8(80 \%)$ & 0.476 \\
\hline Need for ICP Monitor & $58(81 \%)$ & $9(90 \%)$ & 0.469 \\
\hline \multicolumn{4}{|l|}{ Need for blood transfusion } \\
\hline First $4 \mathrm{~h}$ & $14(19 \%)$ & $0(0 \%)$ & 0.126 \\
\hline First $24 \mathrm{~h}$ & $22(31 \%)$ & $2(20 \%)$ & 0.492 \\
\hline During Hospitalization & $46(64 \%)$ & $6(60 \%)$ & 0.811 \\
\hline Hospital LOS, days & 20 [10-35] & $10[5-19]$ & 0.106 \\
\hline ICU LOS, days & $14[6-23]$ & $6[5-16]$ & 0.274 \\
\hline Ventilator Days & $6[3-14]$ & $3[3-10]$ & 0.335 \\
\hline
\end{tabular}

Continuous variables presented as median [interquartile range]. Categorical variables presented as number (percentage). All times are given in hours

Neurosurgical Intervention, defined by craniotomy or ICP monitor insertion. $C T H$ computed tomography scan of the head, ED emergency department, ICP intracranial pressure, $L O S$ length of stay, ICU intensive care unit

required neurosurgical intervention. Specifically, in a subgroup analysis of patients requiring craniotomy or ICP monitor insertion, the majority of Immediate CTH patients were moderately disabled upon hospital discharge while the majority of Delayed CTH patients were severely disabled or dead. Although these differences did not reach statistical significance, a type II error must be considered due to the limited sample size as a result of the single-center nature of this study.

Conceptually, if a patient requires craniotomy for TBI, it is intuitive that that patient's greatest chance for functional recovery lies in prompt surgical decompression. It is for this reason that decompressive craniotomies are not performed days after they are deemed necessary. Much as hospitals may not be expediting the work-up of suspected myocardial infarction if this merely shortened door-to-balloon time instead of reducing mortality and other major cardiac events [18], time to CTH and its impact on outcomes after TBI must be further studied to conclusively show a clinical benefit in terms of functional recovery. The next step in this study will be multicenter evaluation to demonstrate a statistically significant clinical benefit to Immediate $\mathrm{CTH}$.

Our findings also demonstrated that despite similar arrival GCS and Head AIS scores between study groups, patients who underwent Immediate $\mathrm{CTH}$ had shorter times to craniotomy, ICP monitor insertion, ED disposition decision, and ED exit. Although the shortened times to neurosurgical intervention were not statistically significant, these differences may still be clinically relevant as patients with Immediate CTH underwent craniotomy or ICP monitor insertion hours earlier than patients with Delayed CTH. The times to ED disposition decision and exit were significantly abbreviated after Immediate CTH.

The association between expedited CTH and faster times to decision-making and discharge out of the ED add weight to the concept that resource expenditure to improve access to the CT scanner for trauma patients with moderately depressed GCS on arrival to the ED may be beneficial for emergency departments and hospitals [7]. Shortened ED time for these patients may also improve overall ED throughput by allowing an increased number of patients to be assessed.

The study limitations must be considered. In addition to the restricted sample size and possible resultant type II error, this was a retrospective study with functional outcomes assessment based on chart review. It is possible that prospective study with patient interview-based assessment of functional outcomes may have yielded more divergent results. Additionally, post-discharge functional outcomes data were unavailable. Because functional neurologic recovery can certainly evolve over the intermediate to long term, the lack of post-discharge data precludes our ability to comment on the impact of time to first CTH on long-term functional outcomes after trauma. Next, patient comorbidities and home medications, including anticoagulants or antiplatelet agents, were not captured. It is possible that time to CTH has a differential impact on patients who are systemically anticoagulated prior to head trauma and this should be addressed in the future with prospective study. We also did not collect data on the initial pupillary exam. Because the pupillary exam provides additional information about neurologic injury that is not captured by the GCS score, this variable should be included in future study of this subject. Furthermore, in the current study, we focused on functional neurologic recovery and did not measure the behavioral, cognitive, or emotional impacts of TBI. Therefore, these aspects of recovery after head injury are not examined by this study. Lastly, the specific indications for Immediate vs. Delayed imaging could not be discerned given the retrospective observational nature of the study. Improved comprehension of the clinical rationale for the timing of cross-sectional imaging with a prospective study may further clarify the optimal time frame for CTH among patients presenting with moderately depressed GCS after trauma.

In summary, shortened time to CTH among patients with blunt head trauma and first ED GCS of 9-12 was associated with more rapid discharge destination decision from the 
ED. No statistically significant improvement in functional outcomes at discharge was demonstrated based on time to CTH. However, particularly among patients who required neurosurgical intervention, this may have been due to a type II error as a result of the small sample size of this study. The next step in the examination of the impact of time to CTH on functional outcomes after TBI is multicenter examination.

Funding No funding was received for this study.

\section{Declarations}

Conflict of interest Authors Schellenberg, Benjamin, Cowan, Owattanapanich, Wong, Inaba, and Demetriades declare that they have no conflict of interest.

Ethical approval All procedures performed in this retrospective observational study involving human participants were in accordance with the ethical standards of the Institutional Review Board of the University of California (HS-20-00,020) and with the 1964 Helsinki declaration and its later amendments or comparable ethical standards.

Informed consent This study received a waiver for informed consent by the Institutional Review Board of the University of Southern California (HS-20-00,020).

\section{References}

1. American College of Surgeons Committee on Trauma (ACS COT). Resources for optimal care of the injured patient. 6th ed. Chicago, Il: American College of Surgeons; 2014.

2. Bardes JM, Benjamin E, Schellenberg M, Inaba K, Demetriades D. Old age with a traumatic mechanism of injury should be a trauma team activation criterion. J Emerg Med. 2019;57(2):151-5.

3. Schellenberg M, Benjamin E, Bardes JM, Inaba K, Demetriades D. Undertriaged trauma patients: who are we missing? J Trauma. 2019;87(4):865-9.

4. Benjamin ER, Khor D, Cho J, Biswas S, Inaba K, Demetriades D. The age of undertriage: current trauma triage criteria underestimate the role of age and comorbidities in early mortality. J Emerg Med. 2018;55(2):278-87.

5. Polites SF, Leonard JM, Glasgow AE, Zielinski MD, Jenkins DH, Habermann ER. Undertriage after severe injury among united states trauma centers and the impact on mortality. Am J Surg. 2018;216(4):813-8.
6. Tignanelli CJ, Vander Kolk WE, Mikhail JN, Delano MJ, Hemmila MR. Noncompliance with American College of Surgeons Committee on Trauma recommended criteria for full trauma team activation is associated with undertriage deaths. J Trauma. 2018;84(2):287-94.

7. Schellenberg M, Benjamin E, Owattanapanich N, Inaba K, Demetriades D. Impact of delayed time to first CT head in traumatic brain injury. Eur J Trauma Emerg Surg. 2020. https://doi.org/10. 1007/s00068-020-01421-1.

8. Watanitanon A, Lyons VH, Lele AV, Krishnamoorthy V, Chaikittisilpa N, Chandee T, et al. Clinical epidemiology of adults with moderate traumatic brain injury. Crit Care Med. 2018;46(5):781-7.

9. Einarsen CE, van der Naalt J, Jacobs B, Follestad T, Moen KG, Vik A, et al. Moderate traumatic brain injury: clinical characteristics and a prognostic model of 12-month outcome. World Neurosurg. 2018;114:e1199-210.

10. Hoffmann M, Lehmann W, Rueger JM, Lefering R, Trauma Registry of the German Society for Trauma Surgery. Introduction of a novel trauma score. J Trauma. 2012;73(6):1607-13.

11. Marshall SA, Riechers RG 2nd. Diagnosis and management of moderate and severe traumatic brain injury sustained in combat. Mil Med. 2012;177(8 Suppl):76-85.

12. Kim JJ, Gean AD. Imaging for the diagnosis and management of traumatic brain injury. Neurotherapeutics. 2011;8(1):39-53.

13. Gempeler A, Orrego-Gonzalez E, Hernandez-Casanas A, Castro AM, Aristizabal-Mayor JD, Mejia-Mantilla JH. Incidence and effect of diabetes insipidus in the acute care of patients with severe traumatic brain injury. Neurocrit Care. 2020;33(3):718-24.

14. Banks JL, Marotta CA. Outcomes validity and reliability of the modified rankin scale: implications for stroke clinical trials. Stroke. 2007;38(3):1091-6.

15. van Dijck JTJM, Mostert CQB, Greeven APA, Kompanje EJO, Peul WC, de Ruiter GCW, et al. Functional outcome, in-hospital healthcare consumption and in-hospital costs for hospitalised traumatic brain injury patients: a dutch prospective multicentre study. Acta Neurochir (Wien). 2020;162(7):1607-18.

16. Kasner SE. Clinical interpretation and use of stroke scales. Lancet Neurol. 2006;5(7):603-12.

17. Roberts BW, Kilgannon JH, Hunter BR, Puskarich MA, Pierce L, Donnino M, et al. Association between early hyperoxia exposure after resuscitation from cardiac arrest and neurological disability: prospective multicenter protocol-directed cohort study. Circulation. 2018;137(20):2114-24.

18. Soon CY, Chan WX, Tan HC. The impact of time-to-balloon on outcomes in patients undergoing modern primary angioplasty for acute myocardial infarction. Singapore Med J. 2007;48(2):131-6. 